\title{
ARTICLE OPEN \\ Polymer nanofibre composite nonwovens with metal-like electrical conductivity
}

Steffen Reich ${ }^{1}$, Matthias Burgard ${ }^{1}$, Markus Langner ${ }^{1}$, Shaohua Jiang ${ }^{2}$, Xueqin Wang ${ }^{3}$, Seema Agarwal ${ }^{1}$, Bin Ding ${ }^{3,4}$, Jianyong Yu ${ }^{3,4}$ and $^{2}$ Andreas Greiner ${ }^{1}$

Bendable and breathable polymer nanofibre nonwovens with metal-like electrical conductivity are required for lightweight electrodes and electric shielding design with applications in batteries, functional textiles, sensors, cars, aerospace, constructions, mobile phones, and medical devices. Metal-like conductivity in polymer nonwovens has not been achieved till now due to the limitation of the existing processing techniques. We show here, the metal-like electrical conductivity of $750,000 \mathrm{~S} / \mathrm{m}$ in polyacrylonitrile (PAN), poly( $\varepsilon$-caprolactone) $(\mathrm{PCL})$ nonwoven using very low content of silver nanowires (AgNW; 3.35 vol\%). The key to the high conductivity was the homogenous distribution of AgNW in nonwoven made by wet-laid process using short electrospun fibre and AgNW dispersion. Above a threshold of $0.36 \mathrm{vol} \% \mathrm{AgNW}$, the conductivity of the nonwoven increased by seven orders of magnitude, which we attribute to the onset of percolation of the AgNW. Our nonwoven-AgNW composites show fast heating and cooling within a few seconds at a voltage of $1.1 \mathrm{~V}$, which is in the range of portable devices. These composites are also breathable and bendable. The electrical conductivity was independent of the bending angle of the composite, which is important for applications mentioned above and would help other scientists to design new conductive materials in the future.

npj Flexible Electronics (2018)2:5 ; doi:10.1038/s41528-017-0018-5

\section{INTRODUCTION}

Electrically conductive sponges, nonwovens, films and fibres are of major importance for energy production, ${ }^{1}$ energy storage, ${ }^{2}$ sensors ${ }^{3}$ and actuators. ${ }^{4}$ Polymers often form an integral part of nanostructured materials, as they are mechanically flexible, easily processable, have low thermal conductivity, chemical stability, high strength to weight ratio and are lightweight. However, most polymers are also electrically insulating, which limits their applications in electrodes.

Besides, metal coating (aluminium, copper, silver), ${ }^{5,6}$ two primary strategies are used to produce polymer-based materials capable of conducting electricity: the use of conductive polymers (such as polyaniline (PANI), polypyrrole (PPy), polythiophene and polyfluorene), ${ }^{7}$ or the use of polymer composites with silver, copper or gold nanoparticles, ${ }^{6,8}$ metal nanowires ${ }^{9-11}$ or carbon materials as conductive additives. ${ }^{12-15}$ The inherent conductivity, and the amount and shape of the additive are important parameters for the conductivity of the composite. Lower amount of additives with elongated shape in a composite is required for high conductivity as compared to spherical additives. ${ }^{16}$ Lower amount of additive could be favourable for reduced specific weight and mechanical or optical properties. For example, polymer composites with spherical metal particles and carbon black feature decent conductivities at volume fractions of over 5 vol $\%{ }^{17}$ and 20 vol $\%{ }^{18}$ respectively. In contrast, wire shaped additives, such as conductive metallic or carbon nanowires, with high aspect ratios from 100-500, show in films high conductivities even with volume fractions $\leq 1$ vol\%. ${ }^{19}$ Over the past decade, the use of conductive silver nanowires (AgNW) has been investigated for several applications ${ }^{20}$ including organic light-emitting diodes (OLED), ${ }^{13,21}$ solar cells, ${ }^{22}$ touch panels, ${ }^{23}$ wearable electronics, ${ }^{24}$ strain sensors, ${ }^{25}$ piezoelectric energy harvester ${ }^{26}$ and paper-based electronics, ${ }^{27}$ because of the conductivity and transparency of polymer composite films. Nevertheless, the electrical conductivity of AgNW polymer composite films could not reach metal-like conductivity due to the inherent insulation of AgNW by the polymer matrix. Like in polymer films metal-like conductivity was also not achieved in polymer nonwovens. Reason for this is the limited processability of micro- and nanofibre together with AgNW or similar conductive additives. The state-of-the-art method for the preparation of micro- and nanofibre nonwovens is electrospinning. ${ }^{28}$ In electrospinning a materials jet is formed from a droplet of a viscous polymer solution by the action of a strong electrical field. This material jet is pulled continuously towards the counter electrode and deposits as a nanofibre nonwoven. Electrospinning of polymer solutions with suspended conductive additives such as AgNW has been reported but resulted in encapsulation of the AgNW in the nanofibres of the nonwovens. ${ }^{29}$ Consequently, the conductivity of the resulting nonwovens is relatively low and does not exceed $0.2 \mathrm{~S} / \mathrm{m}^{30}$

In the present work, we present a straightforward methodology for the preparation of polymer composite nanofibre nonwovens with metal-like electrical conductivity of up to $750.000 \mathrm{~S} / \mathrm{m}$ at very low content of AgNW (<3.4 vol\%) as conductive additive. These composites are also breathable, bendable and mechanically stable. Key to the metal-like conductivity was the homogenous distribution of long AgNW in the nonwoven and the percolation of the AgNW electrical contacts. The homogenous distribution of

\footnotetext{
${ }^{1}$ Macromolecular Chemistry, Bavarian Polymer Institute, University of Bayreuth, Bayreuth 95440, Germany; ${ }^{2}$ College of Materials Science and Engineering, Nanjing Forestry

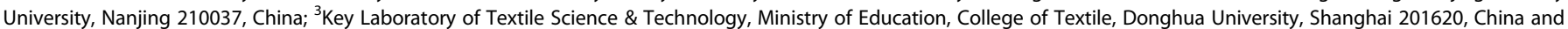
${ }^{4}$ Innovation Center for Textile Science and Technology, Donghua University, Shanghai 20051, China Correspondence: Andreas Greiner (greiner@uni-bayreuth.de)
}

Received: 7 September 2017 Revised: 4 November 2017 Accepted: 23 November 2017

Published online: 14 February 2018 
AgNW in the nonwoven was achieved by the so-called wet-laid method, ${ }^{31}$ which is the formation of a nonwoven by filtration of a fibre suspension in a solvent. Obstacles in the processing of high aspect ratio AgNW with homogenous distribution can be overcome by the utilisation of the wet-laid process. Besides homogenous distribution of high aspect ratio AgNW, other advantages of the wet-laid process are that composite nonwovens could be fabricated by a roll-to-roll process and many other additives could be added for multifunctionality. For the formation of our composite nonwovens we have used in the suspensions for the wet-laid process AgNW in combination with short electrospun PAN and PCL fibres. The electrospun fibres had to be shortened since with as-electrospun fibres no homogenous suspension could be achieved. After moderate annealing of the composite, which was required for the bonding of the composite nonwoven by melting of the PCL fibres, we obtained the nonwovens with metallike conductivity. We discuss in detail the impact of the amount of AgNW on the electrical conductivity and provide a qualitative as well as quantitative explanation based on the percolation theory. We answer the question why a low amount of conductive additive, here AgNW, is sufficient for the achievement of metallike electrical conductivity. We are convinced that our concept will have a broad impact on application in battery electrodes, supercapacitors, functional textiles, sensors, medical implants and as conductive construction parts in cars, aeronautics and mobile phone. Our concept of making metal-like electrically conductive porous nonwovens by wet-laid process in combination with high aspect ratio metal nanowires is highly versatile and could be applied to numerous other materials as well.

\section{RESULTS AND DISCUSSION}

Nonwovens composed of short electrospun PAN nanofibres and $\mathrm{PCL}$ microfibres were prepared with different amounts of AgNW (PAN/PCL/AgNW nonwovens) by the recently established wet-laid process ${ }^{31}$ as illustrated schematically in Fig. 1a and parameters as given in Table S1. The wet-laid process is ideal for the preparation of composite nonwovens as fibre dispersions are used, which has been exploited here for the mixture of electrospun fibres with AgNW. Firstly, dispersions of short electrospun PAN and PCL were prepared by mechanical cutting of the as-electrospun fibres in a solvent mixture of 2-propanol and water (7:3 by volume) followed by the addition of AgNW. PCL served here as glue for the stabilisation of the nonwovens while the AgNW served as conductive additive. Secondly, the composite nonwovens of PAN and PCL fibres and AgNW were wet-laid by filtration of the dispersion on stainless steel sieve. After drying, the nonwovens were stabilised by gentle pressing between two glass plates at $75^{\circ}$ C.

In general, fibrous nonwovens are characterised by the diameter of the fibres, the porosity, and the pore size. The diameter of the electrospun PAN and PCL fibres, respectively ranged from $307 \pm 82 \mathrm{~nm}$ and $714 \pm 593 \mathrm{~nm}$ according to SEM images (Fig. 1b, c). The porosity (78\%) and the pore size $(1.3 \mu \mathrm{m})$ of the wet-laid PAN/PCL nonwovens without AgNW compare well to the porosity $(87 \%)$ and pore size $(3.9 \mu \mathrm{m})$ of the electrospun PAN nonwovens. The length of the AgNW ranged from 5-34 $\mu \mathrm{m}$ and their diameter from $25-150 \mathrm{~nm}$ for batch A (Fig. 1d, Supplementary Fig. S1a-d) and from length of 10-95 $\mu \mathrm{m}$ and a diameter from 27-143 nm for batch B (Fig. S1e). The scanning electron microscopy with backscattered electron detector (SEM/ $\mathrm{BSE}$ ) and energy-dispersive X-ray analysis (EDX) displayed homogenous distribution of the PAN fibres and AgNW in the composite nonwovens obtained by the wet-laid process (Fig. 1f, $g$ and Figs. $\mathrm{S} 2-\mathrm{S} 5)$. The $\mathrm{PCL}$ fibres are no more visible as they were molten during the stabilisation step at $75^{\circ} \mathrm{C}$. The porosity of composite nonwovens PAN/PCL/AgNW increased up to $93 \%$ with increasing amount of AgNW (Tab. S1). A detailed discussion of the nonwoven morphology for all compositions is given in the Supplementary Information. The inherent porosity of the composite nonwovens provide gas flow through the system and thereby breathability. In a qualitative experiment to confirm breathability, the PAN/PCL/ AgNW nonwoven is fixed to one end of a rubber tube. Carbon dioxide gas generated from solid carbon dioxide at room temperature passed through the nonwoven tied at the end of the tube and bubbled through a phenolphthalein/limewater solution and discoloured it (Video S1). The non-porous polyethylene film was used as a negative blank (Video S2). The breathability is controlled by the mean pore size, which was nearly independent of the AgNW content (Fig. S6). AgNW showed a weight loss of about $3 \mathrm{wt} \%$ in thermogravimetric analysis (Fig. S7), which we attribute to the presence of poly(vinyl pyrrolidone) (PVP). PVP was used in the synthesis of AgNW. The content of AgNW in the nonwoven, given as the volume percentage (vol\%), ranged from 0.16 to $3.35 \mathrm{vol} \%$. The content of AgNW was calculated using eqs. S1 and S2 (see Supplementary Information) based on the weight percent (wt\%) of AgNW obtained by thermogravimetric analysis (Fig. S8; ranged from 1.8 to $77.15 \mathrm{wt} \%$ ). The mechanical properties of the composite nonwovens were in the typical range for electrospun nonwoven (Fig. S9). They showed a slight increase in Young's modulus up to about $140 \mathrm{MPa}$ with increasing AgNW content, which was threefold higher compared to the pure wet-laid PAN/PCL composite nonwoven. The elongation at break decreased slightly with increasing amount of AgNW content. The bending stiffness of the composite nonwovens was low and varied insignificantly between 28-90 $\mathrm{mN}$ (Table S2), which is in the typical range of soft electrospun nonwovens. ${ }^{32}$

The electrical conductivity of the composite nonwovens was calculated according to eqs. S3-S5 in the Supplementary Information based on the resistance of the nonwovens (Table S1). Composite nonwoven with the highest electrical conductivity of $750,000 \mathrm{~S} / \mathrm{m}$ was achieved with a AgNW (batch B) content of 3.35 vol\%. This conductivity was five orders of magnitude higher compared to conductive composite nonwovens with an additive content $<10$ vol\% (Fig. 2a). The AgNW with diameter ca. $25 \mathrm{~nm}$ and length up to 5 and $10 \mu \mathrm{m}$ showed percolation threshold in the range of $0.50-0.75$ vol\% for polystyrene composite films. ${ }^{10}$ The electrical conductivity of our composite nonwovens increased with increasing AgNW content with a discontinuous jump between $0.27-0.36$ vol\% AgNW in the composite nonwoven (Fig. $2 b)$. Above 0.36 vol\% the electrical conductivity could be extrapolated as a function of the AgNW content by volume to the electrical conductivity of the pure AgNW $(1,970,000 \mathrm{~S} / \mathrm{m}$, batch A). The conductivity of the composite nonwoven with $0.27 \mathrm{vol} \%$ AgNW was $0.1 \mathrm{~S} / \mathrm{m}$, whereas the composite nonwoven $0.36 \mathrm{vol} \%$ AgNW showed a conductivity of $3050 \mathrm{~S} / \mathrm{m}$. This jump in conductivity by a small increase in AgNW content could be rationalised as the percolation threshold of AgNW throughout the whole sample, as supported by SEM analysis (Fig. 1f, $g$ and Figs. S2 and S3). Qualitative inspection of the SEM images of the nonwovens with 0.36 vol\% or more AgNW clearly shows overlapping of the nanowires (Fig. 1f, g), which is the prerequisite of the percolation for the conductivity. The increase in the conductivity at very low volume content of AgNW in the composite nonwoven can be rationalised by the percolation theory, which predicts that with rod-like additives significantly less amount additive is required as compared to spherical particles in order to achieve property (for example conductivity) percolation in a composite. This prediction has been fully exploited in the concept of our conductive composite nonwovens with AgNW as conductive additive. The number of AgNW in a defined volume can be calculated using eq. (1):

Number of nanowires $=\frac{4 C V}{D_{\mathrm{Ag}} \pi d^{2} L}$ 
a

Fibre dispersion AgNW dispersion
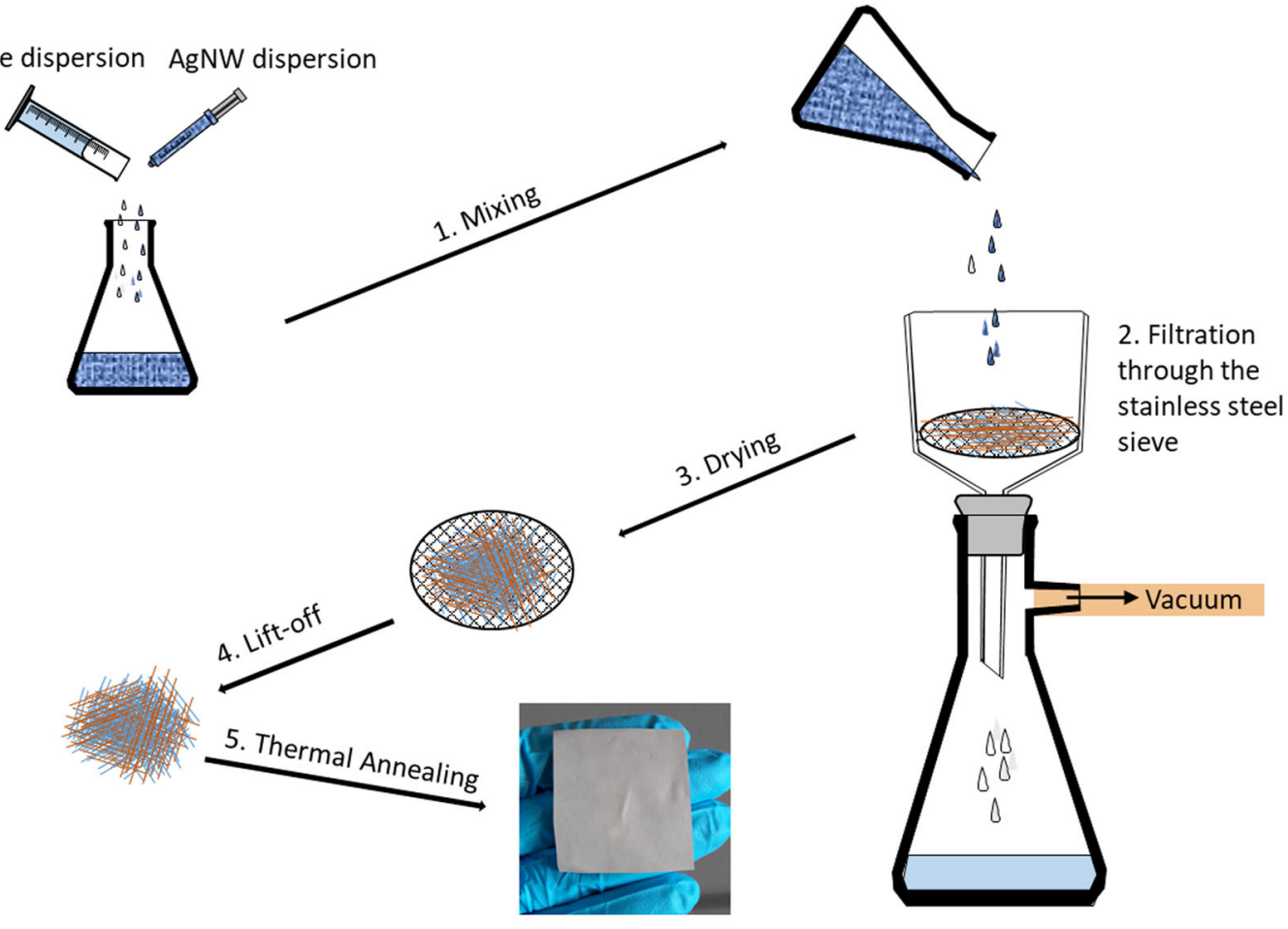

b

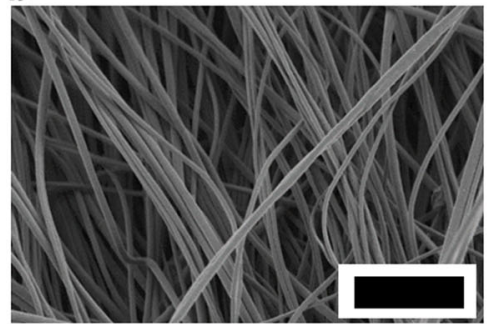

c
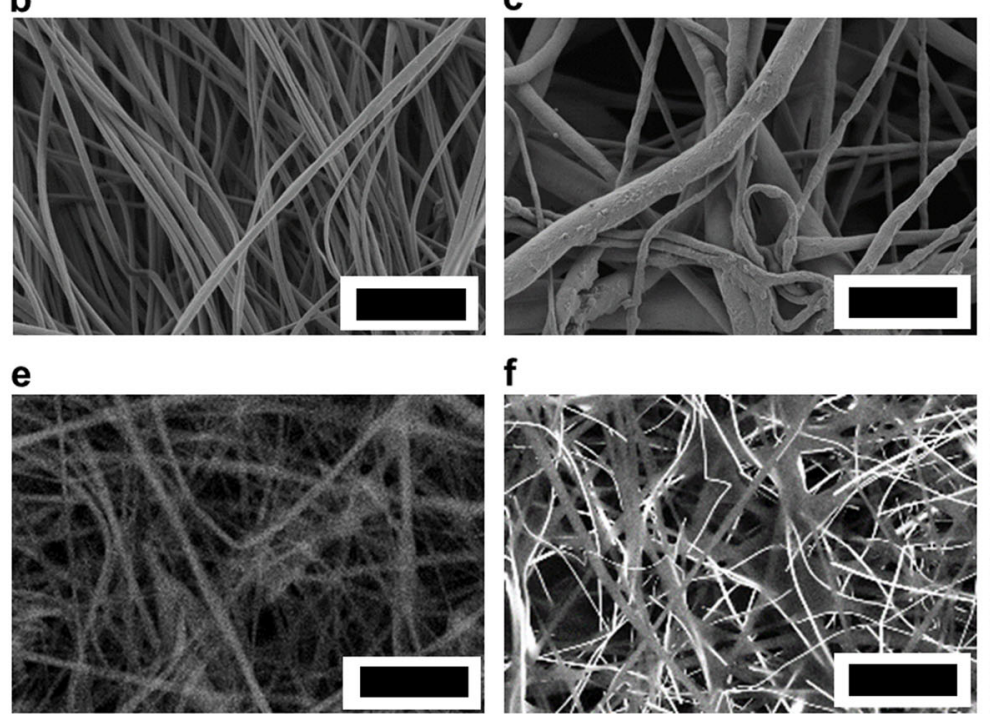

f

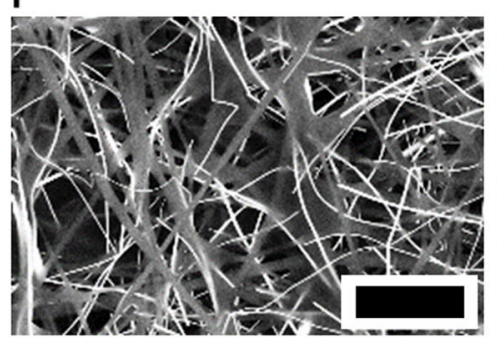

d

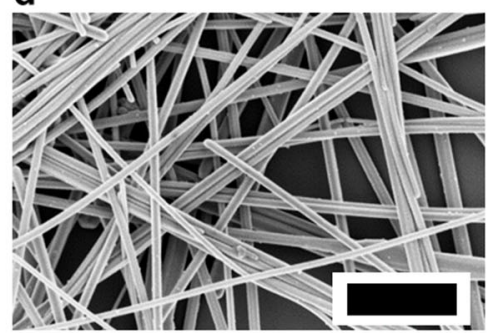

$\mathrm{g}$

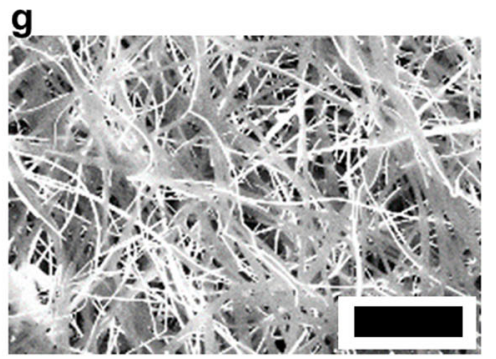

Fig. 1 Preparation and morphology of PAN/PCL/AgNW nonwovens prepared by the wet-laid process. a Schematic of the procedure for the preparation of the PAN/PCL/AgNW nonwovens. (1-5) 1 Mixing of PAN nanofibres, PCL nanofibres and AgNW. 2 Filtration of the PAN/PCL/ AgNW dispersion through the stainless steel sieve. 3 Drying of the PAN/PCL/AgNW nonwoven. 4 Lift-off of the PAN/PCL/AgNW nonwoven from the stainless steel sieve. 5 Thermal annealing of the PAN/PCL/AgNW nonwoven. SEM images of the polymeric and metallic fibres: $\mathbf{b}$ Aselectrospun PAN fibres, $\mathbf{c}$ as-electrospun PCL fibres, $\mathbf{d}$ AgNW; SEM/BSE images of the composite nonwovens with increasing AgNW content: $\mathbf{e}$ 0.00 vol\%, f 0.36 vol\% and $\mathbf{g} 1.07$ vol\%. scale bar $5 \mu \mathrm{m}(\mathbf{d} 1 \mu \mathrm{m})$

where $C$ is the concentration of the AgNW dispersion (183 mg/ $\mathrm{mL}), V$ is the volume of the AgNW dispersion, $D_{\mathrm{Ag}}$ is the density of $\mathrm{Ag}\left(10.5 \mathrm{~g} / \mathrm{cm}^{3}\right), d$ is the diameter $(76 \pm 28 \mathrm{~nm})$ and $L$ is the length of the AgNW (14.24 $\pm 6.27 \mu \mathrm{m})$. The number of AgNW is given $N=$ $0.8476 \mathrm{~V} / \mu \mathrm{m}^{3}$ for the given volume of the AgNW dispersion. In order to describe the correlation of the conductivity quantitatively by the density of the AgNW in our composite nonwovens the value for the critical exponent in Eq. (2) for the dimensionality (a) should be 1.33 (for two dimensional (2D) system) or 1.9 (for three- dimensional (3D) system). ${ }^{33}$

$\sigma \sim\left(N-N_{c}\right)^{a}$

$\sigma$ is the conductivity in $3 D$ and $N_{c}$ is the critical nanowire density for the onset of percolation. $N_{c}$ is obtained by plotting of the sheet resistance versus the volume of the nanowire dispersion (Fig. S10). The relationship between $\sigma$ and the sheet resistance, $R_{\mathrm{sh}}$, is $\sigma=1$ / $R_{\text {sh, }}$ and the relationship between the volume $V$ of the AgNW dispersion and $R_{\mathrm{sh}}$ is given by Eq. (3). From this calculation the 
a
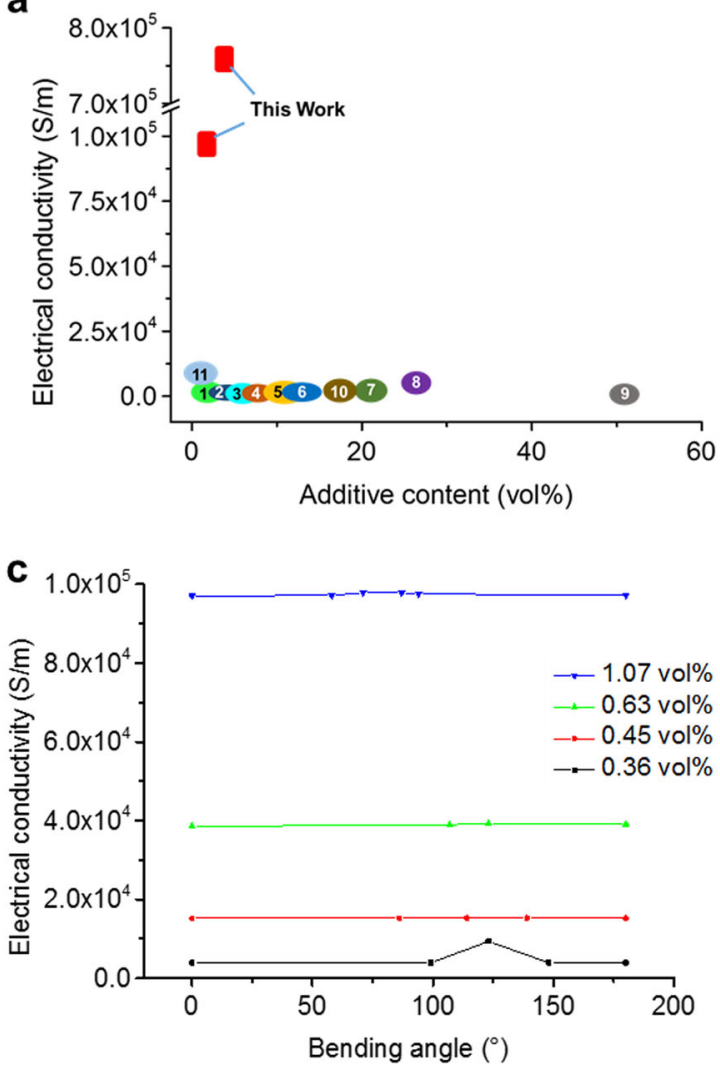

b

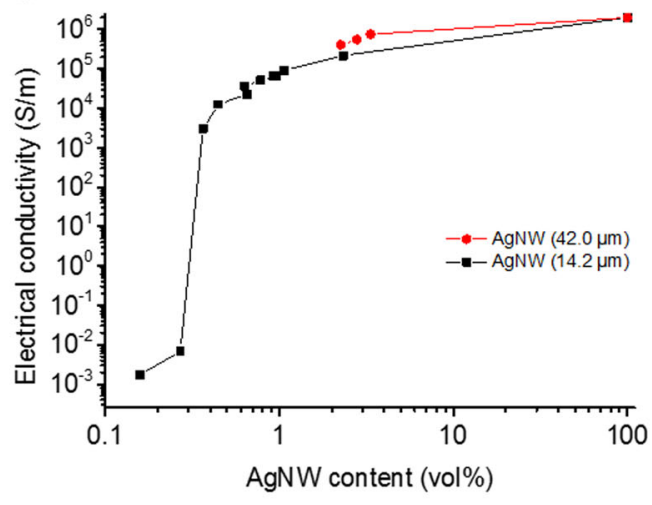

d

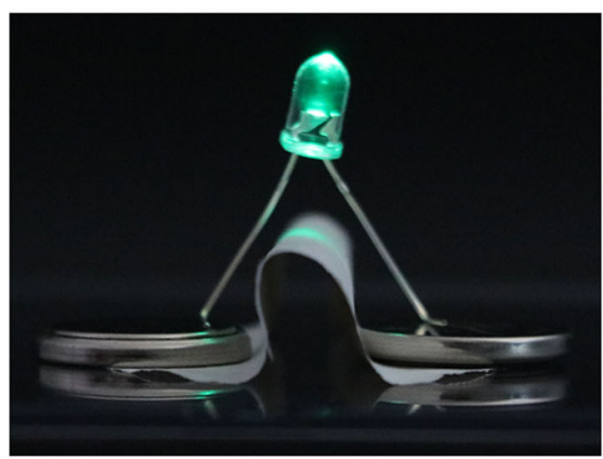

Fig. 2 Electrical properties of conductive nonwovens. a Ashby plot showing the electrical conductivity of different conductive composite nonwovens against their conductive additive content: 1 carbon nanotube/polylactide(PLA) fibres ${ }^{42} ; 2$ carbon black/PLA fibres ${ }^{42}$; 3 PANI/ natural rubber fibres ${ }^{43}$; 4 PANI-dodecylbenzene sulphonic acid/Co-PAN fibres ${ }^{44} ; 5$ carbon nanotube/PET fibres ${ }^{45}$; 6 PANI/bacterial cellulose fibres ${ }^{46} ; 7$ poly(3,4-ethylenedioxythiophene):polystyrene sulphonate (PEDOT:PSS/PNN) fibres ${ }^{47} ; 8$ PEDOT:PSS/hollow carbon fibres ${ }^{48}$; 9 PANI/ polystyrene fibres ${ }^{49} ; 10$ MWNT/cellulose fibres ${ }^{50} ; 11$ AgNW/cellulose fibres. ${ }^{51}$ b Electrical conductivity with respect to the AgNW content. c Electrical conductivity dependence on bending angle. d Photograph of an LED operated by two coin batteries connected by a flexible conductive PAN/PCL nonwoven with 1.07 vol\% AgNW

critical exponent $a$ can be derived (inset of Fig. S10).

$R_{\mathrm{sh}} \sim\left(V-V_{\mathrm{c}}\right)^{-a}$

According to these calculations based on the percolation theory the value for a for our composite nonwovens with AgNW is $1.60 \pm$ 0.07 , which is centred between 1.33 for $2 \mathrm{D}$ systems and 1.9 for $3 \mathrm{D}$ systems. The results obtained for the value $a$ match the morphology of the composite nonwovens, which are neither flat films nor real 3D object but voluminous (porosity up to $93 \%$ ).

The level of conductivity could be almost doubled by use of longer AgNW with an average length of $42.0 \mu \mathrm{m}$ (AgNW batch B, Fig. S1e) as compared to shorter AgNW with average length of $14.2 \mu \mathrm{m}$ (AgNW batch A, Fig. S1d). The electrical conductivity of the composite nonwoven made with shorter AgNW (2.30 vol \%) was about $220,000 \mathrm{~S} / \mathrm{m}$. In contrast, the electrical conductivity was almost doubled to about 407,000 S/m with longer AgNW (batch B) at similar content (2.25 vol\%). The length of the AgNWs plays very important role to achieve superior electrical conductivity. The longer AgNW provide an effective and longer percolation network thereby better conductivity as shown in other literatures. ${ }^{34,35}$

The percolation of the AgNW is obviously not affected by the bending of the composite nonwovens as the conductivity is nearly independent of the bending angle (Fig. 2c). Consequently, the operation of a commercial light-emitting diode by a bended nonwoven with coin batteries was possible (Fig. $2 \mathrm{~d}$ ). The durability of the nonwovens can be demonstrated by bending the nonwoven 10,000 times without changing the electrical conductivity. For example, PAN/PCL/AgNW nonwoven with 0.90 vol\%
AgNW (batch A) after bending for 10,000 times (Video S3) show the electrical conductivity of $106,625 \pm 8347 \mathrm{~S} / \mathrm{m}$, which is comparable to the electrical conductivity of $100,185 \pm 9927 \mathrm{~S} / \mathrm{m}$ before bending. There are several reports about conductive and stretchable conductors ${ }^{20}$ in which conductive metal nanowires are typically deposited on top of substrates, such as poly (ethylene terephthalate) (PET), polydimethylsiloxane (PDMS) and polyacrylates. ${ }^{34,36}$ In such systems, nanowires exist as a network layer on a flexible substrate or embedded in polymer film where just one network layer of the AgNW creates the electrical conductive pathways. The conductivity is decreased after a limited tensile/ compressive strain due to the weakening and detachment of the NW contacts.

Moreover, delamination under repeated mechanical loading could also occur. ${ }^{37,38}$ In our system lot of AgNW layers are over and under each other in the whole bulk of the material and they are not embedded in the polymer. So if the membrane is bended there will be other connections (percolation network) between the silver nanowires created causing almost no change in conductivity.

A very interesting feature of the electrically conductive composite nonwovens is their ability to act as Joule heaters at a constant voltage of $1.1 \mathrm{~V}$, which is in the range of portable devices. The maximum temperature increased at constant voltage of $1.1 \mathrm{~V}$ with the content of AgNW (Fig. 3a). Within $20 \mathrm{~s}$ the composite nonwoven with $1.07 \mathrm{vol} \% \mathrm{AgNW}$ heats up to about $80^{\circ} \mathrm{C}$ and cools down immediately when the voltage is off. The polymerAgNW Joule heaters are known in the literature ${ }^{39}$ and fast heating 
a

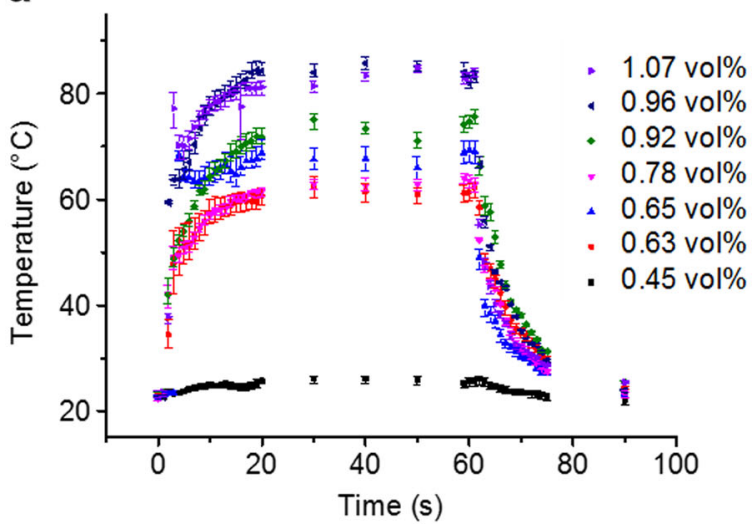

b

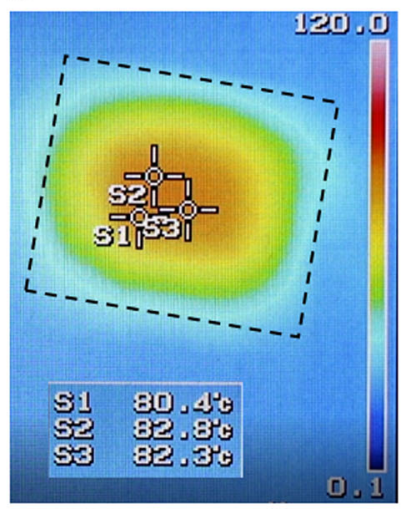

Fig. 3 Heating at constant voltage with increasing AgNW content. a Joule heating at $1.1 \mathrm{~V}$ for nonwovens with different AgNW contents. b IR camera image with an applied voltage of $1.1 \mathrm{~V}$ and 1.07 vol\% AgNW nonwoven material (dotted lines). Scale unit: ${ }^{\circ} \mathrm{C}$

and cooling of our PAN/PCL/AgNW nonwoven compares well with the fastest heating and cooling rates of other AgNW-based films. ${ }^{40}$ The infrared (IR) camera image of the heated sample showed homogenous distribution of temperature with gradual cooling towards the edges of the sample (Fig. 3b). This Joule heating and cooling can be performed for a sample with 0.90 vol\% AgNW (batch $\mathrm{A}$ ) in the bended or unbended state while the temperature for unbended reached $67^{\circ} \mathrm{C}$ and bended reached $61^{\circ} \mathrm{C}$.

The joule heating is shown to affect the deformation of the low glass transition temperature $(\mathrm{Tg})$ polymers, such as polyethylene terephthalate (PET) substrate $\left(\mathrm{Tg} \sim 75^{\circ} \mathrm{C}\right)$ in literature. ${ }^{41}$ In our case, there was no burning or distortion of sample observed even after three cycles of heating and cooling. This might be due to the fact that the maximum temperature did not exceed the $T g$ of PAN, the matrix polymer.

\section{CONCLUSIONS}

Polymer nanofibre composite nonwovens with metal-like electrical conductivity were achieved by exploitation of the versatility of the wet-laid process. This process enabled the homogenous distribution of AgNW in the polymer nanofibre composites with percolation of the electric contacts between the AgNW. As a result the composites show metal-like conductivities at extremely low volume content of the AgNW, which is plausible by the rod-like shape of AgNW and its low percolation threshold. The increase in length of the AgNW increases the electrical conductivity of the composite very significantly. The design of AgNW will provide more opportunities for further increase of the conductivity of the nonwovens. The concept of conductive composite nonwoven preparation should be widely applicable to other nonwovens based on short micro- and nanofibres prepared by the wet-laid process. Consequently, future work will focus on the implementation of other shape-anisotropic conductive additives in wet-laid micro- and nanofibre nonwovens, the translation of the process to 3D shaped objects, and its application as electrodes in textiles, capacitors, batteries, functional textile and as basis for sensor devices.

\section{METHODS}

Methods, including statements of data availability and any associated accession codes and references, are available in the online version of the paper.

\section{Data availability statement}

The authors declare that the data supporting the findings of this study are available within the article (and its Supplementary Information Files)

\section{ACKNOWLEDGEMENTS}

We thank the DFG (Deutsche Forschungsgemeinschaft) for financial support. We would also like to thank Franka Gruschwitz for help fabricating the membranes and performing the material characterisation, Martina Heider of BIMF for supporting the SEM/EDX measurements and Judith Schöbel for the TEM measurements.

\section{AUTHOR CONTRIBUTIONS}

S.R. carried out the synthesis and characterisation of the AgNW; the electrospinning, prepared and characterised the nonwovens and wrote the manuscript. M.B. carried out the tensile tests and SEM/BSE measurements. M.L. carried out the electrospinning, cutting of the fibres and the preparation of the fibre dispersions. S.J. provided a second batch of AgNW and revised the manuscript. S.A. discussed the project and revised the manuscript. H.W., B.D. and J. Y. performed the bending stiffness analysis and discussed the results. A.G. provided the ideas, directed the project and wrote the manuscript.

\section{ADDITIONAL INFORMATION}

Supplementary information accompanies the paper on the npj Flexible Electronics website (https://doi.org/10.1038/s41528-017-0018-5).

Competing interests: The authors declare no competing financial interests.

Publisher's note: Springer Nature remains neutral with regard to jurisdictional claims in published maps and institutional affiliations.

\section{REFERENCES}

1. Chen, S. et al. Electrospun and solution blown three-dimensional carbon fibre nonwovens for application as electrodes in microbial fuel cells. Energy Environ. Sci. 4, 1417-1421 (2011).

2. Arico, A. S., Bruce, P., Scrosati, B., Tarascon, J. M. \& van Schalkwijk, W. Nanostructured materials for advanced energy conversion and storage devices. Nat. Mater. 4, 366-377 (2005).

3. Tee, B. C. -K., Wang, C., Allen, R. \& Bao, Z. An electrically and mechanically selfhealing composite with pressure- and flexion-sensitive properties for electronic skin applications. Nat. Nanotech. 7, 825-832 (2012).

4. Lu, W., Smela, E., Adams, P., Zuccarello, G. \& Mattes, B. R. Development of solid-inhollow electrochemical linear actuators using highly conductive polyaniline. Chem. Mater. 16, 1615-1621 (2004).

5. Lee, H. M. et al. Long-term sustainable aluminium precursor solution for highly conductive thin films on rigid and flexible substrates. ACS Appl. Mater. Interfaces 6, 15480-15487 (2014).

6. Langner, M., Agarwal, S., Baudler, A., Schröder, U. \& Greiner, A. Large multipurpose exceptionally conductive polymer sponges obtained by efficient wetchemical metallization. Adv. Funct. Mater. 25, 6182-6188 (2015).

7. Balint, R., Cassidy, N. J. \& Cartmell, S. H. Conductive polymers: towards a smart biomaterial for tissue engineering. Acta Biomater. 10, 2341-2353 (2014).

8. Janovák, L. \& Dékány, I. Optical properties and electric conductivity of gold nanoparticle-containing, hydrogel-based thin layer composite films obtained by photopolymerization. Appl. Surf. Sci. 256, 2809-2817 (2010). 
9. Bergin, S. M. et al. The effect of nanowire length and diameter on the properties of transparent, conducting nanowire films. Nanoscale 4, 1996-2004 (2012).

10. Gelves, G. A., Lin, B., Sundararaj, U. \& Haber, J. A. Low electrical percolation threshold of silver and copper nanowires in polystyrene composites. Adv. Funct. Mater. 16, 2423-2430 (2006).

11. Gong, S. et al. A wearable and highly sensitive pressure sensor with ultrathin gold nanowires. Nat. Commun. 5, 3132 (2014).

12. Gong, T. et al. Low percolation threshold and balanced electrical and mechanical performances in polypropylene/carbon black composites with a continuous segregated structure. Compos., Part B 99, 348-357 (2016).

13. Zhang, D. et al. Transparent, conductive, and flexible carbon nanotube films and their application in organic light-emitting diodes. Nano Lett. 6, 1880-1886 (2006).

14. Sengupta, R., Bhattacharya, M., Bandyopadhyay, S. \& Bhowmick, A. K. A review on the mechanical and electrical properties of graphite and modified graphite reinforced polymer composites. Prog. Polym. Sci. 36, 638-670 (2011).

15. Stankovich, S. et al. Graphene-based composite materials. Nature 442, 282-286 (2006).

16. Jason, N. N., Ho, M. D. \& Cheng, W. Resistive electronic skin. J. Mater. Chem. C. 5, 5845-5866 (2017).

17. Chan, K. L., Mariatti, M., Lockman, Z. \& Sim, L. C. Effects of the size and filler loading on the properties of copper- and silver-nanoparticle-filled epoxy composites. J. Appl. Polym. Sci. 121, 3145-3152 (2011).

18. Saleem, A., Frormann, L. \& Iqbal, A. Mechanical, thermal and electrical resistivity properties of thermoplastic composites filled with carbon fibres and carbon particles. J. Polym. Res. 14, 121-127 (2007).

19. Gelves, G. A., Al-Saleh, M. H. \& Sundararaj, U. Highly electrically conductive and high performance EMI shielding nanowire/polymer nanocomposites by miscible mixing and precipitation. J. Mater. Chem. 21, 829-836 (2011).

20. Gong, S. \& Cheng, W. One-dimensional nanomaterials for soft electronics. Adv. Electron. Mater. 3, 1600314 (2017).

21. Zeng, X. -Y., Zhang, Q. -K., Yu, R. -M. \& Lu, C. -Z. A new transparent conductor: silver nanowire film buried at the surface of a transparent polymer. Adv. Mater. 22, 4484-4488 (2010).

22. $\mathrm{Hu}$, L. et al. Scalable coating and properties of transparent, flexible, silver nanowire electrodes. ACS Nano 4, 2955-2963 (2010).

23. Lee, J. et al. Very long Ag nanowire synthesis and its application in a highly transparent, conductive and flexible metal electrode touch panel. Nanoscale 4, 6408-6414 (2012)

24. Lee, P. et al. Highly stretchable or transparent conductor fabrication by a hierarchical multiscale hybrid nanocomposite. Adv. Funct. Mater. 24, 5671-5678 (2014).

25. Kim, K. K. et al. Highly sensitive and stretchable multidimensional strain sensor with prestrained anisotropic metal nanowire percolation networks. ACS Nano 15, 5240-5247 (2015).

26. Jeong, C. K. et al. A hyper-stretchable elastic-composite energy harvester. Adv. Mater. 27, 2866-2875 (2015).

27. Santhiago, M., Bettini, J., Araujo, S. R. \& Bufon, C. C. B. Three-dimensional organic conductive networks embedded in paper for flexible and foldable devices. ACS Appl. Mater. Interfaces 8, 10661-10664 (2016).

28. Greiner, A. \& Wendorff, J. H. Electrospinning: a fascinating method for the preparation of ultrathin fibres. Angew. Chem. Int. Ed. 46, 5670-5703 (2007).

29. Song, J. et al. Direct electrospinning of Ag/polyvinylpyrrolidone nanocables. Nanoscale 3, 4966-4971 (2011)

30. Satoungar, M. T., Fattahi, S., Azizi, H., \& Mehrizi, M. K. Electrospinning of polylactic acid/silver nanowire biocomposites: antibacterial and electrical resistivity studies. Polym. Compos. http://onlinelibrary.wiley.com/doi/10.1002/pc.24241/full (2017).

31. Langner, M. \& Greiner, A. Wet-laid meets electrospinning: nonwovens for filtration applications from short electrospun polymer nanofibre dispersions. Macromol. Rapid Commun. 37, 351-355 (2016).

32. Si, Y., Mao, X., Zheng, H., Yu, J. \& Ding, B. Silica nanofibrous membranes with ultrasoftness and enhanced tensile strength for thermal insulation. RSC Adv. 5, 6027-6032 (2015).

33. Madaria, A. R., Kumar, A., Ishikawa, F. N. \& Zhou, C. Uniform, highly conductive, and patterned transparent films of a percolating silver nanowire network on rigid and flexible substrates using a dry transfer technique. Nano Res. 3, 564-573 (2010).

34. Lee, P. et al. Highly stretchable and highly conductive metal electrode by very long metal nanowire percolation network. Adv. Mater. 24, 3326-3332 (2012)

35. Lee, J. H., Lee, P., Lee, D., Lee, S. S. \& Ko, S. H. Large-scale synthesis and characterization of very long silver nanowires via successive multistep growth. Cryst. Growth Des. 12, 5598-5605 (2012).

36. Xu, F. \& Zhu, Y. Highly conductive and stretchable silver nanowire conductors. Adv. Mater. 24, 5117-5122 (2012).

37. $\mathrm{Yu}, \mathrm{Z}$. et al. Highly flexible silver nanowire electrodes for shape-memory polymer light-emitting diodes. Adv. Mater. 23, 664-668 (2011).

38. Yun, S. et al. Compliant silver nanowire-polymer composite electrodes for bistable large strain actuation. Adv. Mater. 24, 1321-1327 (2012).

39. Suh, Y. D. et al. Nanowire reinforced nanoparticle nanocomposite for highly flexible transparent electrodes: borrowing ideas from macrocomposites in steelwire reinforced concrete. J. Mater. Chem. C. 5, 791-798 (2017).

40. Lan, W. et al. Ultraflexible transparent film heater made of Ag nanowire/PVA composite for rapid-response thermotherapy pads. ACS Appl. Mater. Interfaces $\mathbf{9}$, 6644-6651 (2017).

41. Jang, D. -W., Lee, J. -H., Kim, A., Lee, S. -B. \& Hong, S. -G. Self-heating-induced deterioration of electromechanical performance in polymer-supported metal films for flexible electronics. Sci. Rep. 7, 12506 (2017).

42. Li, Y. et al. Tuning of vapor sensing behaviors of eco-friendly conductive polymer composites utilizing ramie fibre. Sens. Actuators, B 221, 1279-1289 (2015).

43. Sukitpaneenit, P., Thanpitcha, T., Sirivat, A., Weder, C. \& Rujiravanit, R. Electrical conductivity and mechanical properties of polyaniline/natural rubber composite fibres. J. Appl. Polym. Sci. 106, 4038-4046 (2007).

44. Jianming, J., Wei, P., Shenglin, Y. \& Guang, L. Electrically conductive PANI-DBSA/ Co-PAN composite fibres prepared by wet spinning. Synth. Met. 149, 181-186 (2005).

45. Li, Z., Luo, G., Wei, F. \& Huang, Y. Microstructure of carbon nanotubes/PET conductive composites fibres and their properties. Compos. Sci. Technol. 66, 1022-1029 (2006)

46. Hu, W., Chen, S., Yang, Z., Liu, L. \& Wang, H. Flexible electrically conductive nanocomposite membrane based on bacterial cellulose and polyaniline. J. Phys. Chem. B. 115, 8453-8457 (2011).

47. Huang, S. -R. et al. Thermoresponsive conductive polymer composite thin film and fibre mat: Crosslinked PEDOT:PSS and P(NIPAAm-Co-NMA) composite. J. Polym. Sci. Part A: Polym. Chem. 54, 1078-1087 (2016).

48. Qu, G. et al. A Fibre supercapacitor with high energy density based on hollow graphene/conducting polymer fibre electrode. Adv. Mater. 28, 3646-3652 (2016).

49. Yin, X. H. et al. Percolation conduction in polymer composites containing polypyrrole coated insulating polymer fibre and conducting polymer. Synth. Met. 96, 367-368 (1995).

50. Imai, M., Akiyama, K., Tanaka, T. \& Sano, E. Highly strong and conductive carbon nanotube/cellulose composite paper. Compos. Sci. Technol. 70, 1564-1570 (2010).

51. Lee, T. -W., Lee, S. -E. \& Jeong, Y. G. Highly effective electromagnetic interference shielding materials based on silver nanowire/cellulose papers. ACS Appl. Mater. Interfaces 8, 13123-13132 (2016).

Open Access This article is licensed under a Creative Commons Attribution 4.0 International License, which permits use, sharing, adaptation, distribution and reproduction in any medium or format, as long as you give appropriate credit to the original author(s) and the source, provide a link to the Creative Commons license, and indicate if changes were made. The images or other third party material in this article are included in the article's Creative Commons license, unless indicated otherwise in a credit line to the material. If material is not included in the article's Creative Commons license and your intended use is not permitted by statutory regulation or exceeds the permitted use, you will need to obtain permission directly from the copyright holder. To view a copy of this license, visit http://creativecommons. org/licenses/by/4.0/.

(c) The Author(s) 2018 